\title{
Emittance balancing technique for the resonant slow extraction from a synchrotron
}

\author{
J. Y. Tang, ${ }^{*}$ L. Liu, Z. Yang, S. X. Fang, and X. L. Guan \\ Institute of High Energy Physics, CAS, Yuquan Road 19B, Beijing 100049, China
}

(Received 10 February 2009; published 5 May 2009)

\begin{abstract}
A new method is proposed to produce identical beam emittances in the transverse phase planes from a severely asymmetric beam extracted from a slow-cycling synchrotron. This is important to obtain a good beam distribution at the irradiation spot when the spot scanning method is used for the treatment in hadron therapy. The method makes use of the beam rotation effect either by a solenoid or by a rotational quadrupole section, referred to as rotator, to produce identical emittances in the two transverse phase planes. It is also helpful for the stabilization of the beam spot at the irradiation point. Simulation studies with an artificially generated beam have been carried out to prove the principle. The method is not only applicable to fixed treatment nozzles but also to rotational gantry nozzles. Both a solenoid and a section of quadrupole rotator can be used, with the former being ideal for proton gantries and the latter for fixed carbon nozzles. There is a practical limit in using room-temperature solenoid due to the high beam rigidity in the case of a carbon beam, for which a superconducting solenoid has to be used. The comparison with other methods and the practical applications of the method are also presented.
\end{abstract}

DOI: 10.1103/PhysRevSTAB.12.050101

PACS numbers: 29.27.Eg, 87.56.bd, 29.20.dk

\section{INTRODUCTION}

When a slow-cycling synchrotron is used for the acceleration of protons or carbon ions, the slow extraction method produces a barlike beam in the horizontal phase space with an emittance much smaller than that in the vertical phase space. Although there are different slow extraction methods, they all peel off particles from the circulating beam in one of the transverse phase planes (here taken as the horizontal one) and thus give similar distributions in the transverse phase planes. Even more, both simulations and measurements as conducted in HIMAC [1] show that the distribution in the horizontal phase space is not symmetric due to the resonant extraction process (see Fig. 1). In addition, both the total and rms emittances in the horizontal phase plane may change significantly during a spill and results in the variation of the beam size at the irradiation spot. Hereafter, the rms emittance is used. This is not good for cancer therapy using a scanning method, especially when a gantry nozzle is in use. Because the gantry is rotated to different angles and the beam is not axially symmetric, the coupling between the two transverse planes will result in different beam sizes at the irradiation spot. Unless the beam is properly matched at the entrance to a rotating gantry, the projected emittances in the two transverse phase planes will increase significantly. Hereafter, for convenience, the bending plane within a gantry is still called the horizontal plane and the other transverse plane the vertical plane.

The emittance increase due to the coupling can be cured by a rotator design which was first suggested by Teng [2]

\footnotetext{
*Corresponding author. tangjy@ihep.ac.cn
}

and explained well in Refs. [3,4], where the rotator optics was also introduced by Sato [5], see Fig. 2. A rotator is a group of quadrupoles that is mechanically rotational around the symmetry axis as a whole and has phase advances of $2 \pi$ in one of the transverse phase planes and $\pi$ in the other, and an optimized design usually is composed of six or seven quadrupoles. However, a rotator for the pur-

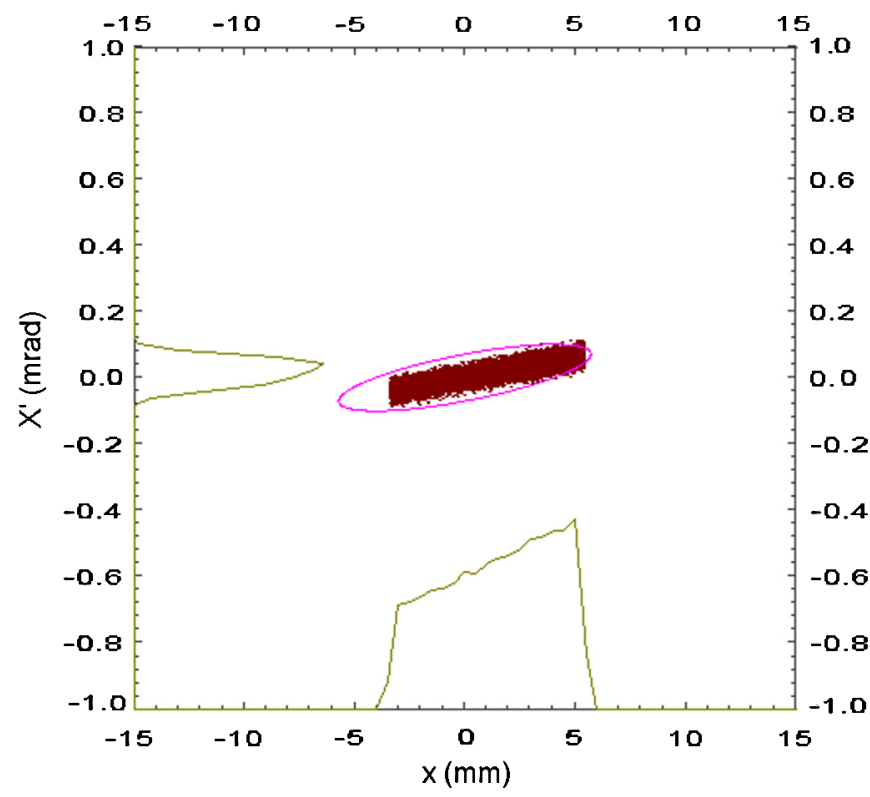

FIG. 1. (Color) Beam distribution in the horizontal phase plane at the entrance of an extraction electrostatic deflector that is artificially generated for simulations. The beam distribution is not symmetric around its center and has a displacement in the position. The fit ellipse is for the matching into the beam transport line. 


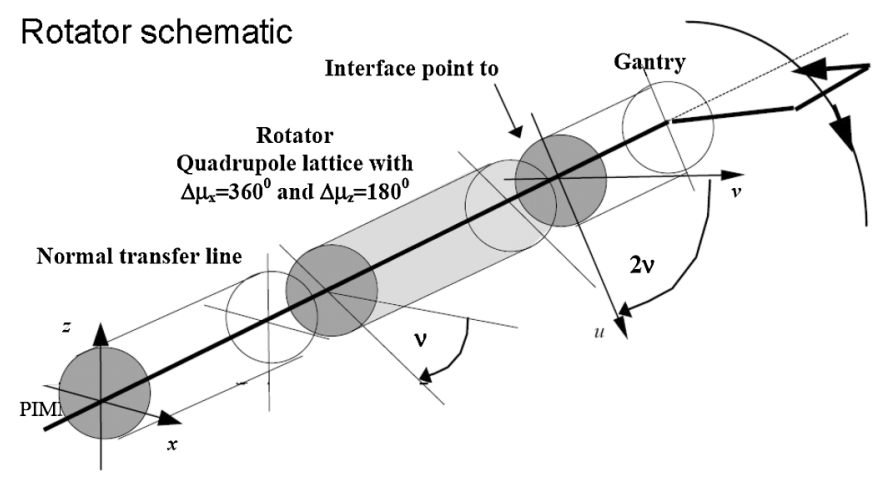

FIG. 2. Schematic for a rotator matched with a gantry (courtesy PIMMS [3]), where the rotation angle of the rotator is half the gantry rotation angle to keep the betatron matching unchanged when the gantry is rotating.

pose of matching to a gantry needs a rotation angle that is half the gantry rotation angle, and it does not solve the problem of the asymmetry in beam emittance. The relatively larger emittance in the gap direction also defines larger magnet sizes and weights of the gantry. To solve the problem of barlike beam orientation in the horizontal plane at different gantries, a sophisticated phase shifter to produce a fixed phase advance between the electrostatic deflector (ESD) at the extraction and any nozzle was suggested in PIMMS (Proton-Ion Medical Machines Study) [4].

With fixed nozzles, a compensation method using a thin scatterer was studied in HIMAC [6], but this method has the drawback of increasing both the horizontal and the vertical emittance. The distribution asymmetry in the horizontal phase plane remains. The scatter thickness should be changed according to the treatment energy, and it also produces local radioactivity. Methods of trying to produce a gantry lattice independent of the rotation angle have been pursued and are proven not flexible to deal with the large ratio between the vertical and horizontal emittances [7].

Here we introduce an emittance balancing technique that will solve the beam asymmetry and stability problems completely.

\section{EMITTANCE BALANCING TECHNIQUE}

\section{A. Principle of emittance balancing technique \\ 1. Emittance balancing using a solenoid}

The method of producing an equal-emittance beam from an asymmetric beam by solenoids is introduced in low energy beam transport lines (LEBT) based on Penningtype $\mathrm{H}^{-}$ion sources in proton linacs [8,9]. There, two or three solenoids are used to make a beam rotation angle of $\pi / 4$ together with producing the required betatron matching.

The transfer matrix $M_{S}$ of the solenoid can be expressed by the product of a rotation matrix $M_{R}$ and a focusing matrix $M_{F}$ :

$$
\begin{aligned}
M_{S} & =M_{R} M_{F} \\
& =\left[\begin{array}{cccc}
C & 0 & S & 0 \\
0 & C & 0 & S \\
-S & 0 & C & 0 \\
0 & -S & 0 & C
\end{array}\right]\left[\begin{array}{cccc}
C & \frac{S}{K} & 0 & 0 \\
-K S & C & 0 & 0 \\
0 & 0 & C & \frac{S}{K} \\
0 & 0 & -K S & C
\end{array}\right],
\end{aligned}
$$

where $C=\cos \theta_{s}=\cos (K L), S=\sin \theta_{s}=\sin (K L)$, and $K=\frac{B_{\|}}{2 B \rho} . B_{\|}$and $B \rho$ are the magnetic field of the solenoid and the beam rigidity. A beam rotation angle by a solenoid, as defined in Eq. (1), also means the Larmor frame with respect to the usual laboratory frame.

If the Courant-Snyder parameters of the input beam are equal in the two transversal planes, namely

$$
\alpha_{x, i}=\alpha_{y, i}, \quad \beta_{x, i}=\beta_{y, i},
$$

then we will obtain

$$
\varepsilon_{x, f}=\varepsilon_{y, f}=\frac{1}{2}\left(\varepsilon_{x, i}+\varepsilon_{y, i}\right) .
$$

Equation (3) has also been approved in the Appendix using an approach different from the one in Ref. [9]. This method can also be applied to beam transport lines for hadron therapy, where the extracted beam from a synchrotron, by using the resonant extraction method, has very different emittances in the horizontal and vertical phase planes.

If only horizontal and vertical fixed nozzles are used, the application of this method is perfect. We just need to insert a solenoid section in the upstream section of the beam transport line, so all the nozzles will have beams with equal emittance. However, when a rotating gantry is involved, the transverse coupling induced by the solenoids will mix with the one induced by the gantry rotation. We have to consider their influence together. Suppose the beam rotation angle by solenoids is $\theta_{s}$ and the rotation angle of the gantry is $\theta_{g}$. The simplest method is to insert a solenoid section just before the gantry entrance. The condition to produce the balanced emittance, as defined by Eq. (3) in the horizontal and vertical planes within the gantry, is

$$
\theta_{T}=\theta_{s}+\theta_{g}=\frac{\pi}{4} \quad \text { or } \quad \frac{3 \pi}{4} .
$$

As mentioned before, here the horizontal plane means the bending plane of the gantry. Because there are no more transverse coupling elements once the beam enters the gantry, the projected emittances in the horizontal and vertical planes remain almost unchanged, except the horizontal one is affected by the dispersion in the gantry. As gantries are usually designed to be achromatic, the equal emittances will be obtained at the irradiation point. Because $\theta_{g}$ is defined by the medical treatment plan, we need to adjust $\theta_{s}$ for different gantry rotation angles.

In a practical application, when we change the beam rotation angle of a solenoid, the focusing strength also changes. This usually is not desired, as we do not want 
to change the transverse matching. Thus, we need to modify the transverse matching section before the solenoid to compensate the focusing effect. Another solution is to divide the solenoid into multiple sections with each field direction being reversible. The combination of different field directions can give the required rotation angle when keeping the focusing unchanged. In this case, spacing is required between the solenoid sections to avoid field interference. It should be mentioned here that the required precision of the rotation angle setting is low, e.g., a few degrees away from the ideal value already gives good results [9].

\section{Emittance balancing using a rotator}

We can also consider using a rotator in the place of a solenoid but in a very different way from that mentioned in Refs. [2-4]. There the rotator rotates the beam following the gantry rotation angle, in order to make the beam matching unchanged when the gantry rotates. Here it is the same as in the case of a solenoid; the rotator and the gantry together will rotate the beam by $\pi / 4$ or $3 \pi / 4$. Thus, we need to compensate only the difference between the gantry rotation angle and $\pi / 4$ (or $3 \pi / 4$ ) by a rotator. Because the beam rotation angle by a rotator is twice its mechanical rotation angle but in a reverse direction, we just need to rotate the rotator by half of the angle difference. It should be mentioned here that the beam rotation by a rotator is not a real rotation expressed by the Larmor rotation frame and it has a difference effect when combined with the beam rotation by a downstream gantry. This is different from a sum effect between the beam rotations by a solenoid and a gantry.

Suppose the rotation angle of the rotator is $\theta_{r}$; the condition to produce equal emittances in the two transverse planes is

$$
\theta_{r}= \begin{cases}\left(\theta_{g}-\frac{\pi}{4}\right) / 2, & \theta_{g}<\frac{\pi}{2} \\ \left(\theta_{g}-\frac{3 \pi}{4}\right) / 2, & \theta_{g} \geq \frac{\pi}{2}\end{cases}
$$

Thus, the rotation range of the rotator is $-\frac{\pi}{8} \leq \theta_{r} \leq \frac{\pi}{8}$. The transfer matrix of the rotator can be expressed by the product of an invertor matrix and a rotation matrix:

$$
\begin{aligned}
M_{\text {rotator }} & =M_{R} M_{\text {invertor }} \\
& =\left[\begin{array}{cccc}
C & 0 & S & 0 \\
0 & C & 0 & S \\
-S & 0 & C & 0 \\
0 & -S & 0 & C
\end{array}\right]\left(\begin{array}{cccc}
1 & 0 & 0 & 0 \\
0 & 1 & 0 & 0 \\
0 & 0 & -1 & 0 \\
0 & 0 & 0 & -1
\end{array}\right) .
\end{aligned}
$$

In the case of following an emittance balancing section, the gantry optics should be designed also with the identical Courant-Snyder parameters in the two transverse planes at the entrance, which is also required by the other gantries using a round beam such as the one extracted from a cyclotron or from a fast cycling synchrotron, but not re-

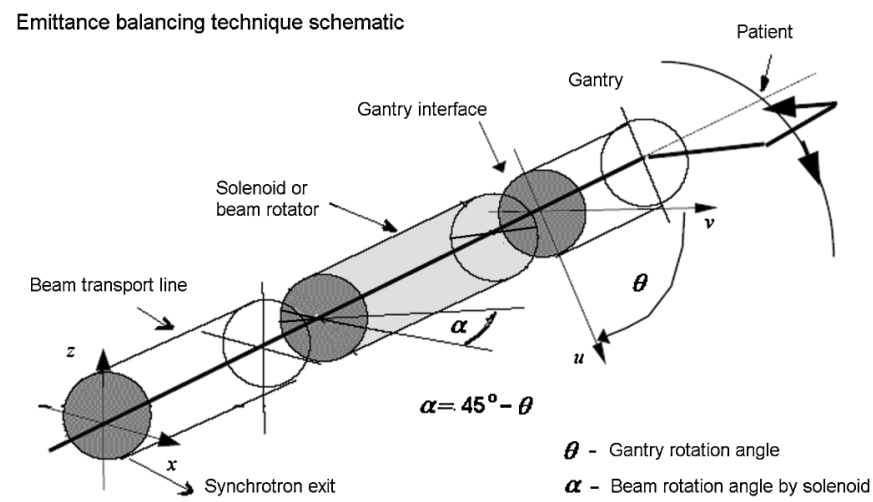

FIG. 3. Schematic for the emittance balancing system matched to a gantry. With a rotator in place, the relation between its rotation angle and the gantry rotation angle should follow Eq. (5).

quired by the gantry following a matching rotator as in Refs. [2-4]. A rotator requires a large installation space; for example, Ref. [3] gives a design of seven quadrupoles with a length of about $9 \mathrm{~m}$, whereas a solenoid section requires only $2.5 \mathrm{~m}$ or so. The matching to a rotator or a solenoid section is very similar (see Fig. 3).

\section{B. Numerical verifications}

The principle of the emittance balancing technique either with solenoids or with a rotator has been confirmed by TRANSPORT [10] calculations. Simulations using TURTLE [11] have also been carried out to show the beam distribution in the phase spaces, especially when different beam distributions are used. The simulation results also support the above principle, as shown in Figs. 4 and 5. The two transverse planes after the emittance balancing will have the same emittance and distribution type. A barlike beam distribution in the horizontal phase plane at the ring extraction is matched to a more equilibrated shape at the entrance of the beam rotation section according to the requirement of equal Courant-Snyder parameters in the two transverse planes. For simplicity, the simulations start from the entrance of the rotation section either with a solenoid or with a rotator.

The initial beam distribution in the horizontal phase plane at the ESD entrance is represented by an artificially generated beam with a linear distribution in $x$ and a Gaussian distribution in $x^{\prime}$ as shown in Fig. 1, whereas the distribution in the vertical phase plane is 2D Gaussian. The emittance in the vertical plane is about 36 times the one in the horizontal plane. The phase advance between the ESD and the entrance of the beam rotation section is found not important in this case. Therefore, the initial distributions are transferred to the entrance of the beam rotation section by an arbitrarily chosen phase advance, here $\pi / 4$ in both of the two transverse planes. A small-aperture gantry similar to the BNL design [12] has been used in the simulations, and a round beam spot at the patient is ob- 


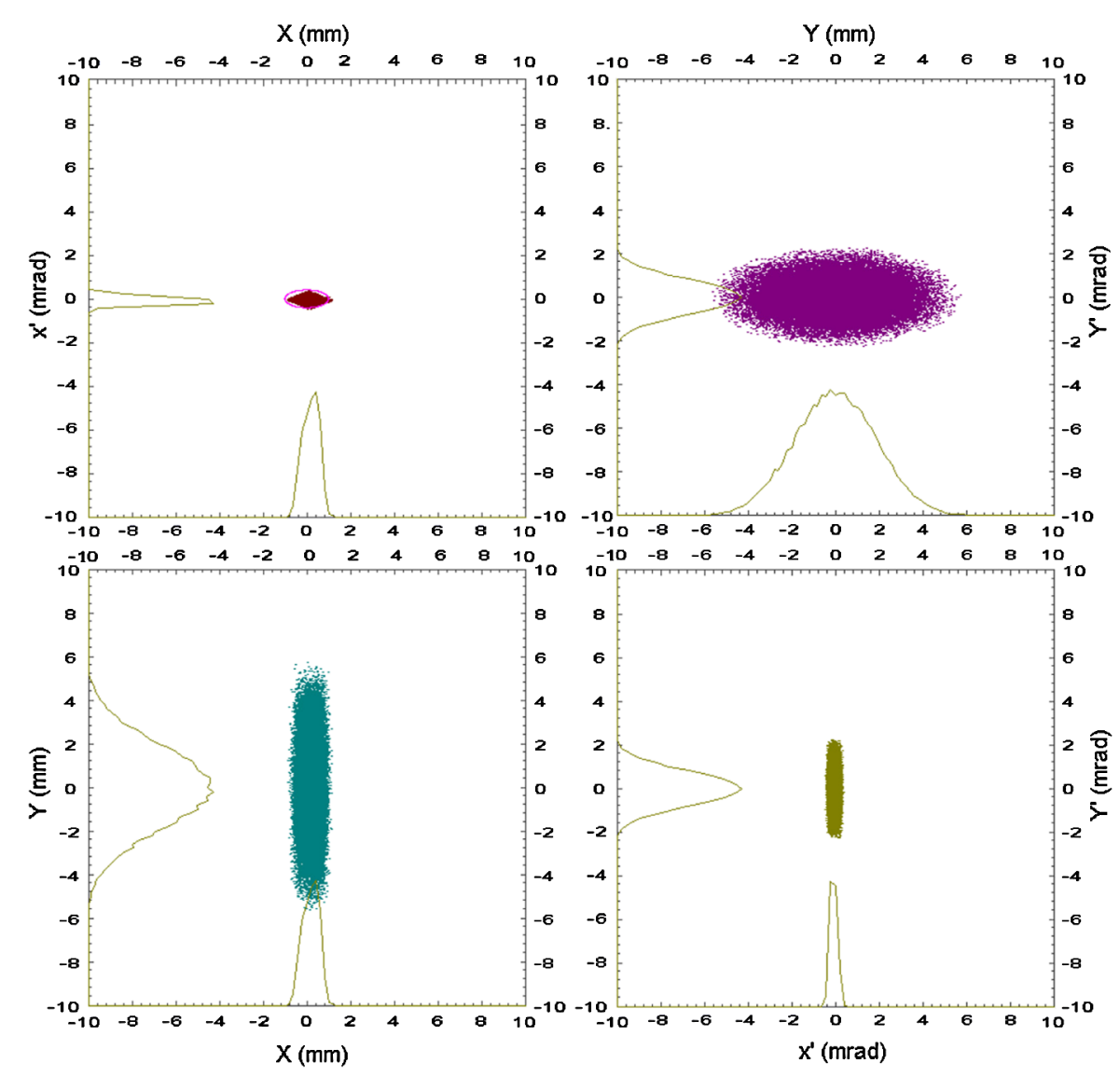

FIG. 4. (Color) Beam distributions in phase spaces at the entrance of the beam rotation section. The initial distributions at the extraction deflector are irregular in the horizontal phase plane and Gaussian in the vertical phase plane, with rms emittances of $0.04 \pi \mathrm{mm}$ mrad and $1.34 \pi \mathrm{mm}$ mrad, respectively. The beam is matched to $\beta_{x}=\beta_{y}=2.48 \mathrm{~m}$ and $\alpha_{x}=\alpha_{y}=0$ at the entrance.

tained by two or three matching quadrupoles in the nozzle section.

The simulation results show that identical emittances are obtained for the two transverse planes in the gantry frame. However, in order to obtain a round beam spot at the irradiation point, we have to impose another constraint $\sigma_{13}=0$ besides the usual envelope matching $\sigma_{11}=\sigma_{33}$ at the spot, where $\sigma$ is the beam matrix. Otherwise, we will see a slanted beam spot as shown in Fig. 5, because the rotation by the solenoids and the gantry itself introduces nonzero coupling terms of the beam matrix that change along the beam line. The correction can be carried out by using the two or three quadrupoles after the last bending magnet in the gantry; these quadrupoles are also used to change the spot size according to the treatment requirement. The nonzero coupling terms such as $\sigma_{32}, \sigma_{41}$, and $\sigma_{42}$ do not pose problems to the treatment spot and are ignored here. This can be explained because within a modest penetration depth in hadron therapy they influence the beam size along the penetration path very little. The phase space plots by TURTLE simulations expose the details of the beam distribution. The statistical results of the simulations with 50000 particles are also shown in
Table I, where one can also see that the beam jitter in the horizontal phase plane is almost overcome by the emittance balancing technique. The gantry angle is set to $20^{\circ}$ from the horizontal plane, and the solenoid strength is matched to the angle.

Because the beam emittance in the horizontal plane is much smaller than in the vertical plane, it contributes very little to the averaged beam emittance. Therefore, the distribution and the Courant-Snyder parameters in the horizontal plane at the entrance of the solenoid section are not critical. This can be seen from Fig. 5, where an initially asymmetric distribution in the horizontal plane does not show up at the irradiation spot. It is the same reason that the original beam center shift in the horizontal plane at the ESD entrance is almost not visible at the final beam spot. One can easily check the arguments by looking through Eq. (A6) in the Appendix. At the entrance of the rotation section, where the coordinates in the horizontal phase plane are usually much smaller than those in the vertical phase plane, therefore, the contribution to the final beam distribution in the form of Courant-Snyder invariant is much less from $I_{x, i}$ and $\left(x_{i}, x_{i}^{\prime}\right)$ compared with that from $I_{y, i}$ and $\left(y_{i}, y_{i}^{\prime}\right)$. The initial beam center shift in the hori- 

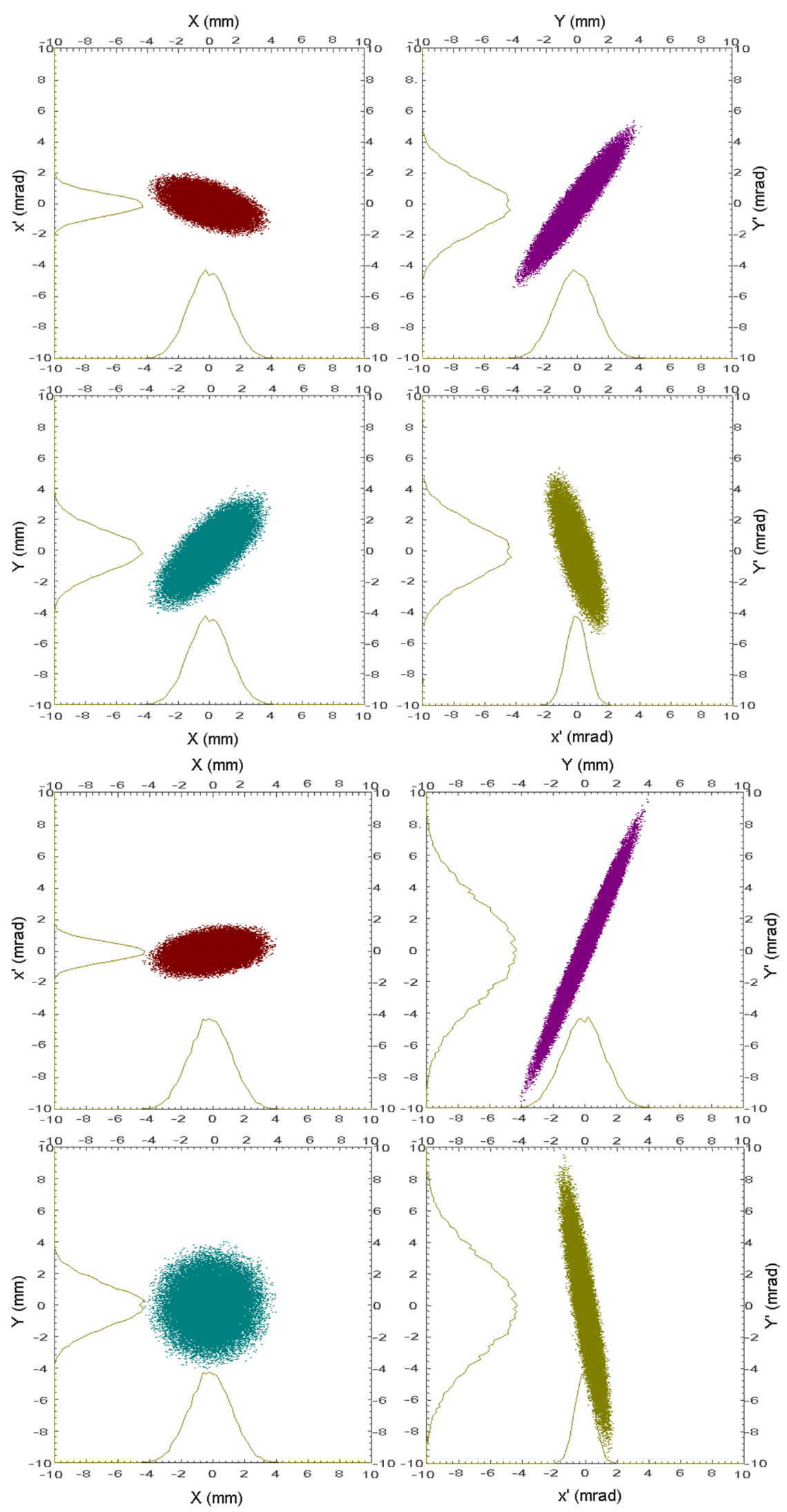

FIG. 5. (Color) Beam distributions in phase spaces at the irradiation spot with a gantry. Upper part: without correction on $\sigma_{13}$, the beam spot in $(X-Y)$ is a slanted ellipse. Lower part: with correction on $\sigma_{13}$ using the three quadrupoles after the last bending magnets, the beam spot is round. In both cases, there is $\beta_{x}=\beta_{y}=2.33 \mathrm{~m}$. 
TABLE I. TURTLE simulation conditions and results with the initial distribution in the horizontal phase plane shown in Fig. 1, where $\varepsilon_{x}, \varepsilon_{y}, \sigma_{x}, \sigma_{y}, x_{c}, x_{c}^{\prime}, y_{c}$, and $y_{c}^{\prime}$ are rms emittance, rms envelopes, and beam centroids. The dispersions are zero at all the locations. The large initial nonzero $x_{c}$ will be transmitted to all elements of the beam matrix when they are correlated. However, the beam centroids become much smaller thanks to the emittance balancing technique.

\begin{tabular}{lcccccccr}
\hline \hline & $\varepsilon_{x}$ & $\varepsilon_{y}$ & $\sigma_{x}$ & $\sigma_{y}$ & $x_{c}$ & $x_{c}^{\prime}$ & $y_{c}$ & $y_{c}^{\prime}$ \\
\hline Position & $\pi \mathrm{mm} \mathrm{mrad}$ & $\pi \mathrm{mm} \mathrm{mrad}$ & $\mathrm{mm}$ & $\mathrm{mm}$ & $\mathrm{mm}$ & $\mathrm{mrad}$ & $\mathrm{mm}$ & $\mathrm{mrad}$ \\
\hline At ESD entrance & 0.04 & 1.33 & 2.90 & 3.65 & 1.48 & 0.02 & 0.00 & 0.00 \\
At solenoid entrance & 0.04 & 1.33 & 0.34 & 1.82 & 0.18 & -0.08 & 0.00 & 0.00 \\
At gantry entrance & 0.69 & 0.69 & 1.38 & 1.17 & -0.17 & -0.04 & -0.05 & -0.05 \\
At irradiation spot & 0.69 & 0.69 & 1.28 & 1.26 & -0.16 & -0.05 & -0.07 & -0.09 \\
\hline \hline
\end{tabular}

zontal phase plane decreases to a very small value at the entrance of the rotation section, because there is a large difference in $\beta_{x}$ between the two locations. One can use Eq. (A4) to see how the centroids are coupled at the beam rotation by replacing coordinates by centroids.

These properties are very helpful in the hadron therapy using a slow-cycling synchrotron, where the extracted beam emittance in the horizontal plane has an irregular distribution and changes largely during a spill and at different extraction energies, and the ripples of the power supplies may result in the beam wobbling at the extraction. After emittance balancing, the two transverse planes will have almost the same distribution shape as the one in the vertical phase space at the extraction, which is often represented by a Gaussian distribution; the horizontal beam wobbling is also reduced largely.

\section{Comparison with other methods}

As mentioned above, the emittance balancing technique will produce an averaged emittance from two much differ- ent emittances in the two transverse planes. There are several advantages with the averaged beam emittance: (1) It is helpful to obtain a round beam at the final irradiation spot. (2) It produces symmetric beam distribution at the spot despite of irregular distribution at the ring extraction. (3) It can reduce the magnet sizes of the gantry due to smaller emittance in the vertical plane. (4) The averaged beam emittance keeps little unchanged when the horizontal beam emittance changes during an extraction spill, because the vertical emittance is dominant. (5) The beam wobbling at the spot due to the ripple during the extraction process is largely reduced. (6) The irregular distribution in the horizontal phase plane at the ring extraction becomes a normal one, the same as the one in the vertical phase plane.

The emittance balancing technique is also compared with other gantry matching methods with a beam extracted from a slow-cycling synchrotron, as shown in Table II. Furthermore, one can say that the averaged emittance with the emittance balancing technique will be comparable with that of a beam extracted from a fast cycling synchro-

TABLE II. Comparison among different matching methods to gantries, where the beam has a large difference between the two transverse emittances. Q denotes quadrupole magnets and B bending magnets.

\begin{tabular}{|c|c|c|c|}
\hline & Beam line & Gantry & Irradiation spot \\
\hline \multirow[t]{2}{*}{ Emittance balancing } & Longer line & Smaller Q magnet sizes & Stable \\
\hline & $\begin{array}{l}\text { Cost solenoids or rotator and } \\
\text { matching } \mathrm{Q}\end{array}$ & $\begin{array}{l}\text { Fixed envelope with respect to (wrt) gantry } \\
\text { angle }\end{array}$ & $\begin{array}{l}\text { Equal and small emittance } \\
\text { Fixed spot Symmetric spot }\end{array}$ \\
\hline \multirow[t]{4}{*}{ Matched rotator } & Longer line & Smaller B magnet sizes & Small $X$ and large $Y$ emittance \\
\hline & Cost rotator and matching $\mathrm{Q}$ & Fixed envelope wrt gantry angle & Fixed spot \\
\hline & Phase shifter in trunk line & & Nonsymmetric spot \\
\hline & & & Less stable \\
\hline \multirow[t]{4}{*}{ Thin scatterer } & Focusing Q in trunk line & Large B and Q magnet sizes & Equal and larger emittance \\
\hline & Variable thickness scatter & Fixed envelope wrt gantry angle & Fixed spot \\
\hline & Local radiation & & Nonsymmetric spot \\
\hline & & & Less stable \\
\hline \multirow[t]{3}{*}{ None } & No cost & Large B and Q magnet sizes & Irregular spot distribution \\
\hline & & Changing envelope wrt & Less stable \\
\hline & & gantry angle & Varying $X$ and $Y$ emittance \\
\hline
\end{tabular}


tron, but significantly smaller than the one of a cyclotron beam.

\section{PRACTICAL APPLICATIONS OF THE EMITTANCE BALANCING TECHNIQUE}

\section{A. Requirements on solenoids and quadrupoles for emittance balancing}

The method of using solenoids is very ideal to produce coupling between the two transverse planes in the case of very low beam energy such as in an LEBT. However, it is less efficient in the case of higher beam energy due to the requirement for stronger field. Here a simple estimation is given about the strength of solenoids used for the emittance balancing technique in a proton therapy facility where the maximum beam energy is $250 \mathrm{MeV}$.

Following the definitions in Eqs. (1) and (4), the maximum required beam rotation angle by a solenoid or solenoids for the emittance balancing technique is

$$
\theta=K L=\frac{B L}{2 B \rho}=\frac{\pi}{4}
$$

Thus, the integration field strength $B L$ of the solenoid is

$$
B L=\frac{\pi}{2} B \rho .
$$

For a proton beam of $250 \mathrm{MeV}$ in energy, $B \rho=2.43 \mathrm{Tm}$, it means an integration field strength of $3.87 \mathrm{Tm}$ for the solenoid, Thus, we need a solenoid of $L=1 \mathrm{~m}$ and $B=$ $3.87 \mathrm{~T}$, for example. This is a typical superconducting solenoid. One can also consider designing a multisection solenoid of $L=2.5 \mathrm{~m}$ and $B=1.55 \mathrm{~T}$ with normal conducting coils. Because of the strong focusing and relatively small emittance, the solenoid aperture is small, and this helps the technical design.

If we consider the carbon therapy, where a $\mathrm{C}^{6+}$ beam of $400 \mathrm{MeV} / \mathrm{u}$ at maximum is used and the beam rigidity reaches $6.35 \mathrm{Tm}$, one has to use superconducting solenoids. For example, a solenoid of $L=2.0 \mathrm{~m}$ and $B=$ $4.99 \mathrm{~T}$ can be considered.

When a rotator section composed of quadrupoles is used for the emittance balancing, the optimized design suggested by Benedikt [3] can be implemented, using normal quadrupoles but with a larger aperture than the other quadrupoles in the delivery beam line. The increased cost comes from the mechanical rotation system and the increased space requirement.

\section{B. Beam rotation in a trunk beam line}

We can design an emittance balancing section-either a rotator or a multisection solenoid-just after the extraction matching section from a synchrotron. The emittance balancing in a trunk beam line will benefit all the treatment nozzles in different treatment rooms. When the beam is directed to a fixed nozzle, the rotation angle is set to $\pi / 4$ for a solenoid and $\pi / 8$ for a rotator. When the beam is directed to a gantry, the rotation angle is set to an angle dependent on the gantry angle, and the phase advances between the rotation-section end and the gantry entrance in the horizontal phase plane should be $\pi$ or its multipliers. The parameters of the quadrupoles or solenoid will change in the same manner as the other magnets when the beam energy changes.

For many carbon therapy facilities without gantries, this seems to be a promising method, as either a superconducting solenoid or a quadrupole rotator with a fixed beam rotation angle of $\pi / 4$ can be used. The latter is favored, because the quadrupoles in the rotator section are just standard skew quadrupoles and no complicated mechanical rotation system is needed.

\section{Beam rotation just before the entrances to gantries}

When a beam rotation section is placed just before the entrance of a gantry, its application is more straightforward despite a higher cost in the case of more than one gantry. There is no matching requirement for the phase advance as required for the method of placing the beam rotation section in the trunk line. In addition to matching the Courant-Snyder parameters at the gantry entrance, one has to also produce identical Courant-Snyder parameters at the entrance of the beam rotation section, which is either a solenoid or a quadrupole rotator. In the case of a solenoid, when the rotation angle by the solenoid changes according to the rotation angle of the gantry, the Courant-Snyder parameters at the solenoid entrance have to be changed but kept identical in the two transverse planes, because the focusing strength of the solenoid also changes. In the case of a quadrupole rotator, the Courant-Snyder parameters at its entrance will be kept the same when the gantry is rotated to a different angle.

\section{A practical design for the beam delivery system}

Here the emittance balancing technique is applied to the practical design of a beam delivery system consisting of three fixed nozzles and one gantry for a proton therapy facility, as shown in Fig. 6. In this facility, there are three treatment rooms. Room A is equipped with a horizontal nozzle, Room B with a horizontal nozzle and a vertical nozzle sharing a treatment couch, and Room $\mathrm{C}$ with a gantry nozzle. It is easy to add more treatment rooms along the trunk line in the future. A solenoid section in the trunk beam line can produce a beam of equalized emittances in the two transverse planes for all the three fixed nozzles: two horizontal nozzles and one vertical nozzle. When the beam is directed towards the gantry, the two parts of the solenoid section are set to be in the opposite polarity to cancel the beam rotation but keep the focusing unchanged. An independent solenoid section just before the gantry produces the required beam rotation. It has been shown 


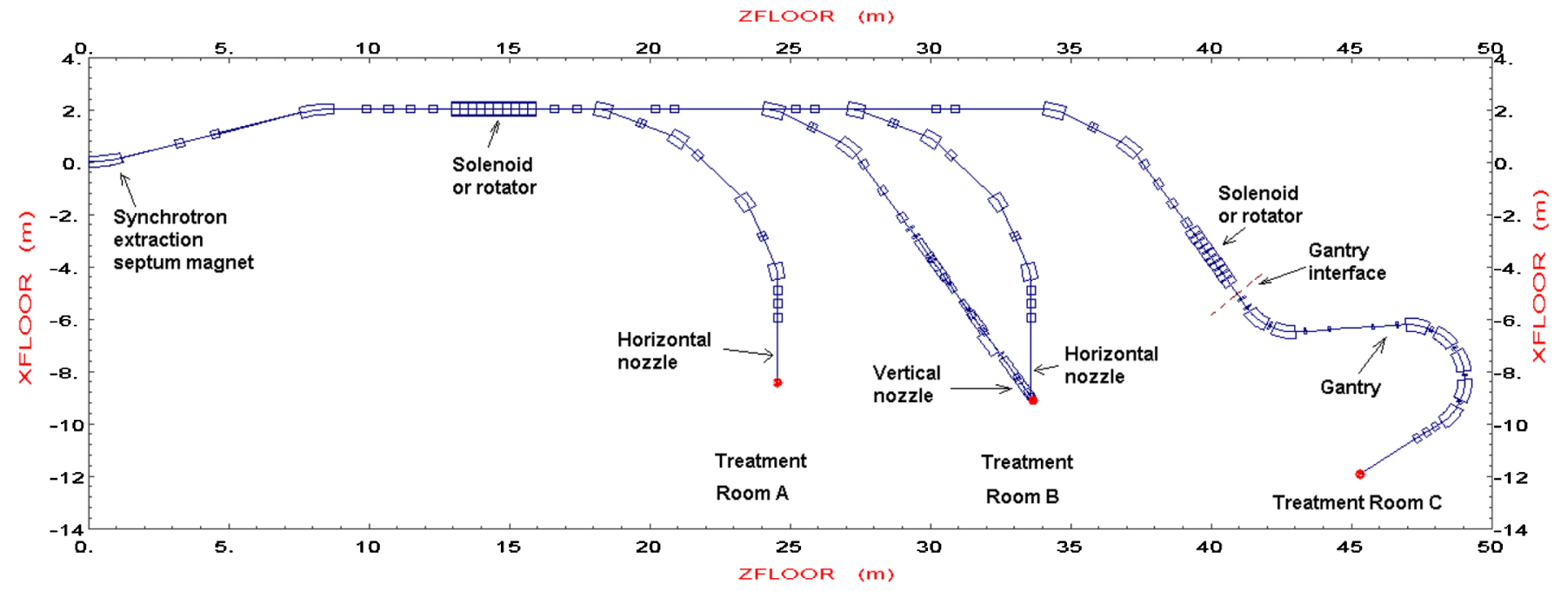

FIG. 6. (Color) An example of beam transport and delivery system for proton therapy using the emittance balancing technique.

that four matching quadrupoles are sufficient to carry out the matching on the Courant-Snyder parameters at the entrance of the solenoid when the solenoid strength changes following the gantry angle.

\section{CONCLUSIONS AND DISCUSSION}

A new method is proposed to produce identical beam emittances in the transverse phase planes from a severely asymmetric beam extracted from a slow-cycling synchrotron, and has been proven by multiparticle simulations. It is important to obtain a good beam distribution at the irradiation spot when the spot scanning method is used for the treatment in hadron therapy. The method is not only applicable to fixed treatment nozzles but also to rotational gantries. Both a solenoid and a section of quadrupole rotator can be used; different application scenarios have been discussed.

The emittance balancing technique primarily shows great potential for spot scanning beam treatments. It also appears to be useful during treatments using methods like wobbling and double scattering where the emittance blowup by scattering is sensitive to the beam spot size at the scatterer. In addition, the technique seems to be extendable to other nonmedical applications where the resonant extraction from a synchrotron is used and a high quality beam spot at target is required.

\section{ACKNOWLEDGMENTS}

This work was partially supported by the National Natural Science Foundation of China (10775153). The authors would like to thank all the members of the IHEP proton therapy study team for their support and discussions, and A. W. Chao from SLAC for the comments and suggestions.

\section{APPENDIX A: PROOF OF EMITTANCE BALANCING BY A BEAM ROTATION}

To achieve the emittance balancing by using a beam rotation, the Courant-Snyder parameters in the two transverse phase planes should be equal. Suppose

$$
\alpha_{x}=\alpha_{y}=\alpha, \quad \beta_{x}=\beta_{y}=\beta, \quad \gamma_{x}=\gamma_{y}=\gamma
$$

For the beam transfer matrix of a solenoid in Eq. (1), with a beam rotation angle of $\pi / 4$ or $3 \pi / 4$, one has

$$
S=\sin \frac{\pi}{4}=a, \quad C= \pm a, \quad a^{2}=\frac{1}{2} .
$$

Thus, the transformation in the coordinates in the phase space of a particle by the rotation matrix is like the following:

$$
\left(\begin{array}{l}
x_{f} \\
x_{f}^{\prime} \\
y_{f} \\
y_{f}^{\prime}
\end{array}\right)=\left(\begin{array}{cccc} 
\pm a & 0 & a & 0 \\
0 & \pm a & 0 & a \\
-a & 0 & \pm a & 0 \\
0 & -a & 0 & \pm a
\end{array}\right)\left(\begin{array}{c}
x_{i} \\
x_{i}^{\prime} \\
y_{i} \\
y_{i}^{\prime}
\end{array}\right)
$$

where the suffixes $i$ and $f$ are for the coordinates of a particle in the phase space before and after the transformation. In the following, the horizontal phase plane and $C=$ $a$ are taken for the further derivations. It is easy to obtain the same results for the vertical phase plane and $C=-a$. One can rewrite Eq. (A3) as

$$
\begin{gathered}
x_{f}=a x_{i}+a y_{i} \quad x_{f}^{\prime}=a x_{i}^{\prime}+a y_{i}^{\prime} \\
y_{f}=-a x_{i}+a y_{i} \quad y_{f}^{\prime}=-a x_{i}^{\prime}+a y^{\prime} .
\end{gathered}
$$

One can use the Courant-Snyder invariant to express the 
deviation of a particle from the reference particle

$$
\begin{aligned}
& I_{x, i}=\gamma x_{i}^{2}+2 \alpha x_{i} x_{i}^{\prime}+\beta x_{i}^{\prime 2} \\
& I_{y, i}=\gamma y_{i}^{2}+2 \alpha y_{i} y_{i}^{\prime}+\beta y_{i}^{\prime 2} .
\end{aligned}
$$

After applying the action of the rotation matrix to the particle, one has

$$
\begin{aligned}
I_{x, f}= & \gamma x_{f}^{2}+2 \alpha x_{f} x_{f}^{\prime}+\beta x_{f}^{\prime 2} \\
= & \gamma a^{2}\left(x_{i}^{2}+2 x_{i} y_{i}+y_{i}^{2}\right) \\
& +2 \alpha a^{2}\left(x_{i} x_{i}^{\prime}+x_{i} y_{i}^{\prime}+x_{i}^{\prime} y_{i}+y_{i} y_{i}^{\prime}\right) \\
& +\beta a^{2}\left(x_{i}^{\prime 2}+2 x_{i}^{\prime} y_{i}^{\prime}+y_{i}^{\prime 2}\right) \\
= & \frac{1}{2}\left(I_{x, i}+I_{y, i}\right)+\left(\gamma x_{i} y_{i}+\alpha x_{i} y_{i}^{\prime}+\alpha x_{i}^{\prime} y_{i}+\beta x_{i}^{\prime} y_{i}^{\prime}\right) .
\end{aligned}
$$

According to Ref. [13], for any kind of distribution one has a relationship between the rms emittance and the statistical averaged invariant as follows:

$$
\varepsilon_{x}=\frac{\bar{I}_{x}}{2\left(1+2 \alpha^{2}\right)}=\frac{1}{2\left(1+2 \alpha^{2}\right)} \frac{1}{N} \sum_{n=1}^{N} I_{x, n},
$$

where $N$ is the total number of the particles. Because the emittances in the two transverse phase planes are initially decorrelated, one has

$$
\begin{array}{rlrl}
\sum_{n=1}^{N} x_{i} y_{i} & =0, & & \sum_{n=1}^{N} x_{i} y_{i}^{\prime}=0, \\
\sum_{n=1}^{N} x_{i}^{\prime} y_{i}=0, & & \sum_{n=1}^{N} x_{i}^{\prime} y_{i}^{\prime}=0 .
\end{array}
$$

By combining Eqs. (A6)-(A8), one has

$$
\varepsilon_{x, f}=\frac{1}{2}\left(\varepsilon_{x, i}+\varepsilon_{y, i}\right)
$$

It is the same for $\varepsilon_{y, f}$. This means that the emittances after the rotation will be the averaged value over the two initial ones.

[1] T. Furukawa et al., in Proceedings of the 9th European Particle Accelerator Conference, Lucerne, 2004 (EPSAG, Lucerne, 2004), pp. 267-269.

[2] L.C. Teng (private communication); Internal Report No. LL-134, 1986.

[3] M. Benedikt and C. Carli, in Proceedings of the Particle Accelerator Conference, Vancouver, BC, Canada, 1997 (IEEE, New York, 1997), p. 1379.

[4] M. Benedikt, P. Bryant, and M. Pullia, Nucl. Instrum. Methods Phys. Res., Sect. A 430, 523 (1999).

[5] S. Kato, Nucl. Instrum. Methods Phys. Res., Sect. A 254, 487 (1987).

[6] T. Furukawa and K. Noda, Nucl. Instrum. Methods Phys. Res., Sect. A 565, 430 (2006).

[7] M. Pavlovic, Nucl. Instrum. Methods Phys. Res., Sect. A 438, 548 (1999).

[8] C. W. Planner, Part. Accel. 48, 243 (1995).

[9] J. H. Li and J. Y. Tang, Nucl. Instrum. Methods Phys. Res., Sect. A 574, 221 (2007).

[10] D. C. Carey, K. L. Brown, and F. Rothacker, Reports No. SLAC-R-530, No. Fermilab-Pub-98-310, and No. UC-414, 1998.

[11] U. Rohrer, PSI Graphic Turtle Framework based on a CERN-SLAC-FERMILAB version by K. L. Brown et al., 2006

[12] S. Peggs et al., in Proceedings of the Particle Accelerator Conference, Chicago, IL, 2001 (IEEE, New York, 2001), pp. 2482-2484.

[13] J. Y. Tang, Nucl. Instrum. Methods Phys. Res., Sect. A 595, 561 (2008). 